\title{
Measuring pulmonary function in COPD using quantitative chest computed tomography analysis
}

\author{
Jens T. Bakker $\mathbb{1}^{1}$, Karin Klooster ${ }^{1}$, Rozemarijn Vliegenthart ${ }^{2}$ and Dirk-Jan Slebos $\mathbb{C}^{1}$ \\ ${ }^{1}$ Dept of Pulmonary Diseases, University of Groningen, University Medical Center Groningen, Groningen, The Netherlands. ${ }^{2}$ Dept of \\ Radiology, University of Groningen, University Medical Center Groningen, Groningen, The Netherlands.
}

Corresponding author: Jens T. Bakker (j.t.bakker@umcg.nl)

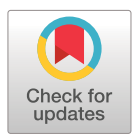

Copyright @The authors 2021
This version is distributed under
the terms of the Creative
Commons Attribution Non-
Commercial Licence 4.0. For
commercial reproduction rights
and permissions contact
permissions@ersnet.org
Received: 8 Feb 2021
Accepted: 8 April 2021
Shareable abstract (@ERSpublications)
CT may be used as a proxy for lung function in COPD diagnosis and evaluation, particularly for the
hyperinflation markers https://bit.ly/2RrGAZf

Cite this article as: Bakker JT, Klooster K, Vliegenthart R, et al. Measuring pulmonary function in COPD using quantitative chest computed tomography analysis. Eur Respir Rev 2021; 30: 210031 [DOI: 10.1183/16000617.0031-2021].

\section{Abstract}

COPD is diagnosed and evaluated by pulmonary function testing (PFT). Chest computed tomography (CT) primarily serves a descriptive role for diagnosis and severity evaluation. CT densitometry-based emphysema quantification and lobar fissure integrity assessment are most commonly used, mainly for lung volume reduction purposes and scientific efforts.

A shift towards a more quantitative role for CT to assess pulmonary function is a logical next step, since more, currently underutilised, information is present in CT images. For instance, lung volumes such as residual volume and total lung capacity can be extracted from CT; these are strongly correlated to lung volumes measured by PFT.

This review assesses the current evidence for use of quantitative CT as a proxy for PFT in COPD and discusses challenges in the movement towards CT as a more quantitative modality in COPD diagnosis and evaluation. To better understand the relevance of the traditional PFT measurements and the role CT might play in the replacement of these parameters, COPD pathology and traditional PFT measurements are discussed.

\section{Introduction}

COPD is a pulmonary disease mainly caused by cigarette smoking, and is the third leading cause of death worldwide [1]. In COPD, exposure to noxious gases causes airway and lung tissue inflammation, leading to small airway resistance increase and lung parenchymal destruction (emphysema).

COPD patients are limited in their expiratory capacity, due to increased resistance of the airways, as well as the reduced elastic recoil of the lung tissue due to emphysema. This causes air trapping and results in hyperinflation. Due to hyperinflation, the respiratory muscles are placed at a mechanical disadvantage for effective inspiration [2]. This reduces the overall capacity for respiration, resulting in dyspnoea.

The main current diagnostic parameters are related to this pathophysiology, using pulmonary function testing (PFT) [3]. Forced expiratory volume in $1 \mathrm{~s}\left(\mathrm{FEV}_{1}\right)$ serves as the primary criterion in COPD diagnosis when related to expiratory vital capacity (VC). $\mathrm{FEV}_{1}$ is a dynamic parameter indicative of obstruction, meaning reduced capacity for expiratory airflow, thus referring to a combination of reduced elastic recoil and increased airway resistance. Total lung capacity (TLC) and residual volume (RV) are lung volumes that characterise the amount of lung overinflation and air trapping. The RV/TLC ratio is associated with mortality [4], which indicates its relevance. In COPD, gas exchange is mainly hindered by diffusion capacity and ventilation/perfusion mismatch [5].

Computed tomography (CT) may enable improvement of COPD diagnostics by using the detailed spatial information available in CT scan images. This review aims to explore the possibility of chest CT-derived 
biomarkers as a proxy for measuring PFT. That is, for patients that already undergo a CT scan for descriptive and/or some quantitative evaluation.

One of the most relevant examples for use of quantitative CT, related to COPD, is in evaluation for treatment by surgical or bronchoscopic lung volume reduction (LVR). For selected emphysema patients with severe hyperinflation, LVR can be an effective treatment option [6]; the most affected parts of the emphysemateous lung are removed or collapsed, thereby reducing the disabling hyperinflation to a more physiological situation [7]. Prior to potential LVR, CT densitometry-based emphysema quantification is performed for lobar selection, and CT-determined fissure integrity evaluation is performed to assess the possibility of collateral ventilation [8, 9]. Traditional PFT measurements, including plethysmography, spirometry and diffusing capacity, are used for further assessment. Even in this area, where the use of quantitative CT is prominent, CT is mainly used in a supportive role to PFT.

However, CT is a modality that is able to capture the tissue densities of the entire chest. Thereby allowing evaluation of lobar emphysema type, and assessment of bronchial wall thickness, beyond the usual PFT results. Clearly, the potential of CT is currently underutilised.

For some of the currently used dynamic parameters, such as $\mathrm{FEV}_{1}$ and diffusing capacity of the lung for carbon monoxide $\left(D_{\mathrm{LCO}}\right)$, it remains unclear if similar information can be derived from static CT images. They lack a clear analogous measurement in the static images provided by CT. The important measures RV and TLC do have clear analogous CT measurements. Therefore, these measurements are expected to be the first to be matched by CT and are the main focus of this review. We summarise the current relevant PFT techniques, review the evidence for measuring PFT parameters by CT, and discuss further development regarding CT-derived PFT measurements in COPD.

\section{Search strategy and selection criteria}

A PubMed search was performed using the terms "computed tomography" or "CT" and "Plethysmography" or "pulmonary function test" and "volumes" or "volume" or "Total lung capacity". The exact search query was: ("computed tomography" [tiab] or CT [tiab]) and (plethysmography [tiab] or "pulmonary function test" [tiab]) and (volumes [tiab] or volume [tiab] or "Total lung capacity" [tiab] or tlc [tiab]). This search query produced 345 results on 6 October 2020. The results were filtered for articles that compared either TLC and/or RV measured by plethysmography to lung volumes derived by CT, with 14 results remaining. These 14 results were further filtered for relevancy by two readers (J.T. Bakker and D-J. Slebos) and the bibliography of these articles was scanned for other articles that were deemed relevant. Relevancy consisted of a correlation between plethysmography and CT derived lung volumes in COPD patients. 10 articles met all relevancy criteria (table 1). No restrictions on the date of publication were used. Articles published in English were exclusively included.

\section{Current methods for measuring lung volumes}

PFT is most often performed according to the guidelines of the European Respiratory Society/American Thoracic Society [20]. In COPD patients, body plethysmography is commonly used in obtaining RV and TLC. Plethysmography derives lung volumes through determination of the intrathoracic gas volume (ITGV). This differs from the functional residual capacity (FRC), used in gas dilution methods, in that it includes all compressible air within the thorax [21], whereas the FRC only includes air that is not trapped.

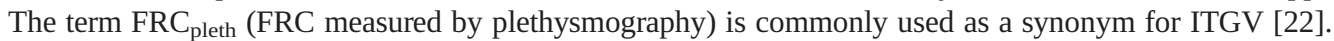

In plethysmography, the TLC and RV are derived by addition and subtraction of the dynamic lung volumes acquired (inspiratory capacity and expiratory reserve volume) with spirometry to the ITGV. For derivation of the ITGV, the patient breathes a small volume against a closed valve back and forth, known as the panting manoeuvre. Measuring the change in alveolar pressure following a small lung volume change derives the ITGV, under the assumption that the mouth pressure equals the alveolar pressure. A greater pressure change signifies a smaller ITGV and vice versa [23]. Since the ITGV measured in plethysmography includes trapped air, this method provides trustworthy data even in COPD patients.

The panting manoeuvre is normally immediately followed by the VC manoeuvre to establish the dynamic volumes. A VC manoeuvre consists of complete expiration followed by complete inspiration. These volumes are measured by means of spirometry and correspond to ERV and IC respectively and together form the VC. A VC manoeuvre immediately following the panting manoeuvre is referred to as the linked method. Figure 1 provides a graphical overview of the different volumes, as well as the relationships between the different volumes. 


\begin{tabular}{|c|c|c|c|c|c|c|c|c|c|}
\hline $\begin{array}{l}\text { First author } \\
\text { [ref.] }\end{array}$ & $\begin{array}{l}\text { Patients } \\
n\end{array}$ & $\underset{n}{\text { COPD }}$ & $\mathrm{FEV}_{1}^{\#}$ & $\begin{array}{c}\mathrm{CT} \\
\text { modules }^{+}\end{array}$ & $\begin{array}{l}\text { Spirometry } \\
\text { gated }\end{array}$ & Software & TLC $R^{2}$ & $\mathrm{RV} \mathrm{R}^{2}$ & VC $R^{2}$ \\
\hline Tantucci [10] & 37 & 20 & $\begin{array}{c}1.16 \pm 0.63 \mathrm{~L} \\
(46.9 \pm 20.6 \%)\end{array}$ & One & Yes & $\begin{array}{c}\text { Aquarius iNtuition, TeraRecon Inc. (Foster } \\
\text { City, CA, USA) }\end{array}$ & 0.97 & & \\
\hline GaRfIELD [11] & 59 & 59 & $\begin{array}{c}1.08 \pm 0.52 \mathrm{~L} \\
(41 \pm 18 \%)\end{array}$ & Multiple & No & $\begin{array}{l}\text { Pulmonary Workstation Plus, VIDA } \\
\text { Diagnostics, Inc. (Coralville, IA, USA) }\end{array}$ & 0.92 & & \\
\hline BECKER [12] & 28 & 28 & $\begin{array}{l}\text { Pre: } 22 \pm 1 \% \\
\text { post: } 29 \pm 2 \%\end{array}$ & One & No & $\begin{array}{c}\text { Custom Visual C ++ (Microsoft; Redmond, } \\
\text { WA, USA) }\end{array}$ & 0.9 & 0.84 & 0.48 \\
\hline Coxson [13] & 57 & 57 & $\begin{array}{l}0.84 \pm 0.23 \mathrm{~L} \\
\text { (all } \leqslant 45 \%)\end{array}$ & Multiple & No & $\begin{array}{c}\text { Custom (EmphylxJ; Vancouver, BC, } \\
\text { Canada) }\end{array}$ & 0.79 & & \\
\hline MAтsumoto [14] & 118 & 46 & & multiple & No & $\begin{array}{c}\text { Semi-automated 'Computer Aided Lung } \\
\text { Informatics for Pathology Evaluation and } \\
\text { Rating' CALIPER }\end{array}$ & 0.96 & & \\
\hline BRown [15] & 486 & 486 & & Multiple & No & $\begin{array}{l}\text { Automated three-dimensional technique } \\
\text { (no further information given) }\end{array}$ & 0.86 & 0.67 & \\
\hline KAUCZOR [16] & 72 & 25 & & One & No & $\begin{array}{c}\text { Allegro workstation (ISG Technologies; } \\
\text { Mississauga, ON, Canada) }\end{array}$ & 0.89 & 0.81 & 0.7 \\
\hline ZAPOROZHAN [17] & 31 & 31 & $35 \pm 11 \%$ & One & No & Custom (YACTA; Mainz, Germany) & 0.9 & 0.83 & \\
\hline BRown [18] & 43 & 4 & & Multiple & No & $\begin{array}{l}\text { Custom (detailed description of } \\
\text { algorithm provided) }\end{array}$ & 0.91 & & \\
\hline Song [19] & 172 & 172 & $\begin{array}{c}0.767 \pm 0.213 \mathrm{~L} \\
(27.9 \pm 4.6 \%)\end{array}$ & One & No & $\begin{array}{l}\text { PULMO3D software (v3.7.1, Fraunhofer } \\
\text { MEVIS; Bremen, Germany) }\end{array}$ & 0.81 & 0.66 & \\
\hline
\end{tabular}

Patients not included in the COPD group suffer from other pulmonary diseases. FEV ${ }_{1}$ : forced expiratory volume in $1 \mathrm{~s}$; TLC: total lung capacity; RV: residual volume; VC: vital capacity. "\#: FEV 1 data were not always available or not available for the COPD group specifically; ": pre and post refer to preoperative and postoperative, respectively; ${ }^{+}$: a set of parameters for a CT scan.

Challenges with plethysmography measurement

Several challenges exist regarding plethysmography, particularly in COPD patients. COPD patients are prone to dyspnoea, which can complicate the measurements. Patients experiencing severe dyspnoea may experience substantial dyspnoea in performing the panting manoeuvre. Dyspnoea results in an inaccurate VC manoeuvre when using the linked method [22]. This method may, therefore, be inadequate for some COPD patients. The unlinked method, where the ITGV and dynamic lung volumes are determined separately, may be prone to inaccuracy due to a possible shift in the ITGV [24]. A difference in plethysmography measurements found between hospitals may be explained by the use of the linked versus the unlinked method $[25,26]$.

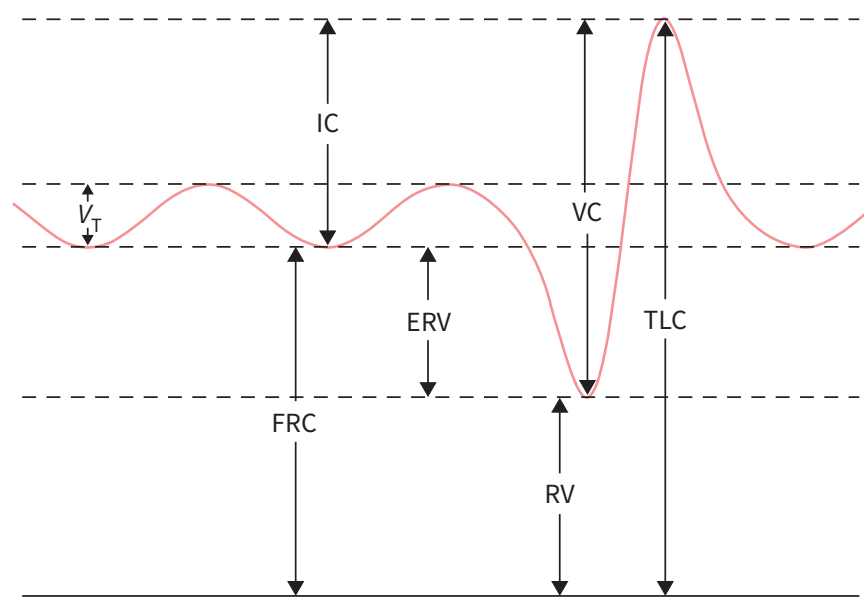

FIGURE 1 Lung volume measurements that are commonly derived from pulmonary function tests. $V_{\mathrm{T}}$ : tidal volume; ERV: expiratory reserve volume; FRC: functional residual capacity; IC: inspiratory capacity; TLC: total lung capacity; RV: residual volume; VC: vital capacity. 
In plethysmography the assumption is made that sudden changes in pressure measured at the mouth are the same as changes in alveolar pressure. In COPD patients, however, this assumption may fail because the airflow obstruction can delay such rapid pressure equilibrium. To prevent overestimation of the TLC, due to a smaller pressure change in the mouth than in alveolar pressure, panting frequency is required to be around $1 \mathrm{~Hz}$ [27].

Furthermore, plethysmography requires frequent calibration to prevent inaccurate measurements [21, 22]. An accurate plethysmography measurement requires two out of three measurements to be within $10 \%$ of each other [23]. Additionally, plethysmography is not as readily available worldwide as other PFT modules, such as spirometry and gas dilution methods [28].

\section{Chest CT scan protocol for PFT measurements}

Chest CT scanning might pose as a good alternative to plethysmography, since one can derive RV and TLC from CT. In addition to the inspiration scan, expiration scans are becoming more prevalent (important for measuring RV).

A reference dose of $650 \mathrm{mGY} \cdot \mathrm{cm}^{-1}$ has been suggested by the European community [29]. In an effort to minimise radiation, suggestions are that the $\mathrm{kVp}$ should be adjusted to the patient size $(80-120 \mathrm{kV})$ and the tube current should be $<250 \mathrm{~mA}$ [30]. Multiple acquisitions are not justified according to the as low as reasonably achievable (ALARA) principle for radiation dose [31].

Furthermore, since COPD patients experience difficulty with expiration and inspiration, scan time will have to be minimised; with newer CT systems the rotation time of the CT system is $<1 \mathrm{~s}$ [30].

The CT acquisition suitable for COPD patients consists of chest high-resolution CT, which was originally intended to provide high image quality for a descriptive evaluation of lung parenchyma. For a descriptive evaluation CT does not necessarily need to adhere to strict protocols. However, when CT is used increasingly quantitatively, standardisation will become increasingly important. Since CT acquisition and reconstruction parameters influence the quantifications directly and thereby indirectly influence the ability to compare outcomes.

Image reconstruction in CT is a trade-off between sharp, noisy images or smooth, less noisy images. Naturally, reconstruction parameters have an influence on quantifications obtained from the images [32]. Different reconstruction and kernel settings are to be preferred for visual versus quantitative evaluation.

\section{Lung segmentation in $\mathrm{CT}$}

Accurate lung segmentation is required to be able to derive lung volumes from CT images. Several approaches for segmentation of the lungs exist [33]. According to MANsoor et al. [34] methods can be grouped into five different classes. These consist of thresholding-based, region-based, shape-based, neighbouring anatomy-guided and machine learning-based methods. Simpler methods, such as thresholding-based methods, are especially prone to failure when there is pulmonary pathology present. However, these are computationally less expensive. More complex models, such as machine learning-based methods, handle abnormalities due to pathology better, but they are generally computationally more expensive. Algorithms often use multiple method classes together to optimally balance accuracy and computational expenditure [34-36]. Figure 2 shows an example of lung segmentation. The algorithm used to segment the lungs may influence the results. This could compromise verification of the results. However, a prior study found strong agreement in segmentation between different algorithms, signified by a correlation factor of 0.995 when comparing volumes found by two algorithms [37].

\section{RV and TLC measured by CT}

With respect to lung volumes based on CT scans, ChONG et al. [38] demonstrated that volumes derived from breath-holding CT scans, scanned 1 week apart, are reproducible. Several studies have found diverse correlations between lung volumes obtained through plethysmography and volumes obtained by CT [10-19, 37, 39-42]. Table 1 shows the subset of these studies that included COPD patients in their study cohort.

Garfield et al. [11], Coxson et al. [13], Мatsumoto et al. [14] and Brown and co-workers [15, 18] included multiple CT parameter settings in their studies, meaning that scan parameters were not the same for each patient. As previously mentioned, not standardising CT settings, manufacturers and reconstruction kernels probably has a negative impact on correlation. The software used by Mатsumoto et al. [14] normalised different CT settings, thereby mitigating the negative impact on correlation, which is reflected in the strong correlations they found. 


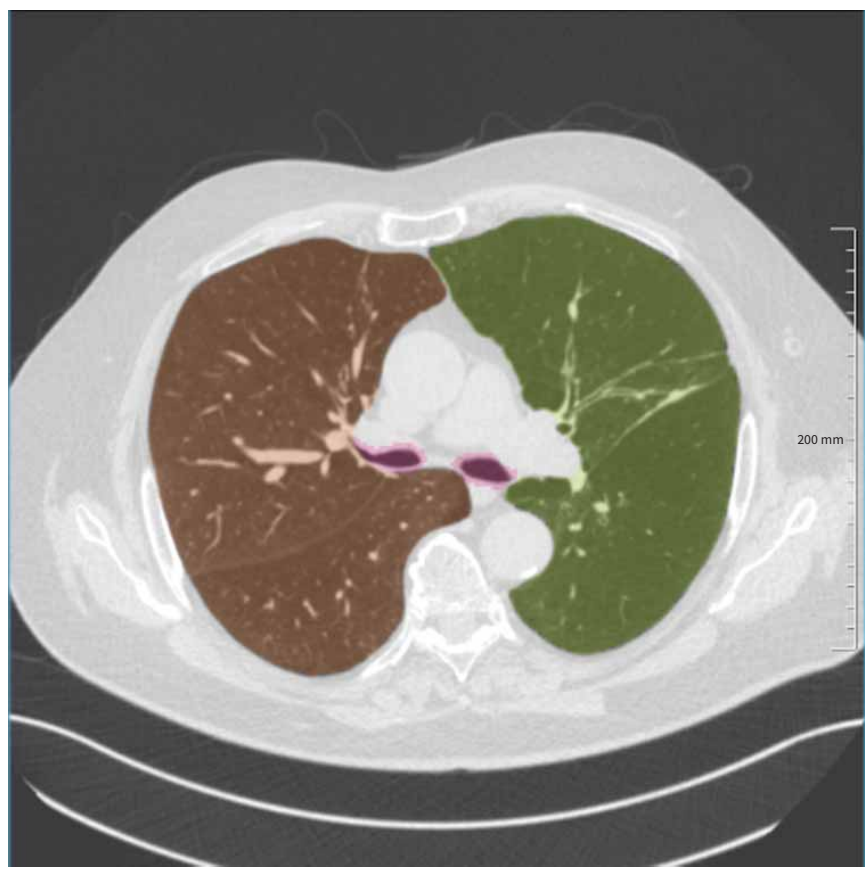

FIGURE 2 Example of chest computed tomography (CT) lung segmentation. The figure shows an axial CT slice at the main carina level. The brown segmentation is the right lung, the green segmentation is the left lung and the purple segmentation shows the large airways (reconstruction made using LungQ, Thirona, Nijmegen, The Netherlands).

Not all studies found the same correlation coefficients or the same regression trends. Results could differ due to a variety of factors. These factors include difference in CT settings and difference in segmentation algorithms and, additionally, plethysmography-derived volumes may differ between hospitals, possibly using the linked method instead of the unlinked method [25]. As previously mentioned, in COPD especially, reaching optimal in- and expiration is an important factor as well.

Generally, TLC measured by plethysmography yielded a higher value than derived by CT, the difference typically being around $0.5-1 \mathrm{~L}$. For RV, the situation is reversed, such that CT yielded a higher value than plethysmography, with the difference ranging from $0.3-1 \mathrm{~L}$. Slight differences between the absolute values derived through CT and plethysmography are expected due to differences in patient positioning. That is, seated in plethysmography versus supine in CT [43-45]. YAmAda et al. [41] compared CT-derived volumes in seated, standing and supine positions and found significant differences $(+10 \%$ and $+9.5 \%$ average increase in seated versus supine for TLC and RV, respectively).

Additionally, inaccuracies in complete in- and expiration are to be expected in CT. The lower TLC measured in CT compared to plethysmography might be caused due to more air being included in the plethysmography measurement. For instance, air in the oesophagus and trachea. This is also true for the RV measurement. However, the therefore seemingly paradoxically higher CT-derived RV is likely due to the population included. These consisted of COPD patients in many studies. These patients have trouble with exhaling completely. In PFT the airflow is carefully monitored, which increases the likelihood of measuring the realistic complete expiration.

Additionally, CT-derived volumes include lung tissue, whereas plethysmography only includes air. This may artificially inflate the volumes derived by CT. RV may be relatively more affected by the artificial inflation than TLC, because tissue occupies a relatively larger proportion in this volume measurement.

Expected benefits from spirometry gating

Successful CT-based lung volume derivation is dependent on the performance of full in- and expiration, the duration of the scan and the synchronisation of these events. By using spirometry gating to time the CT acquisition, the moment of full in- and expiration is monitored, and the CT can be made accordingly. Expectations are that spirometry gating will improve the quality of the volumes derived from CT scans. 
a)

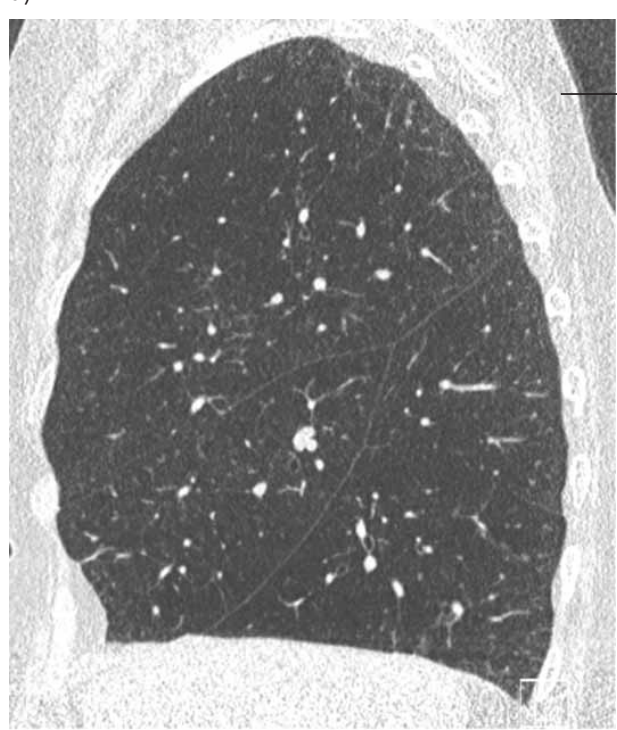

b)

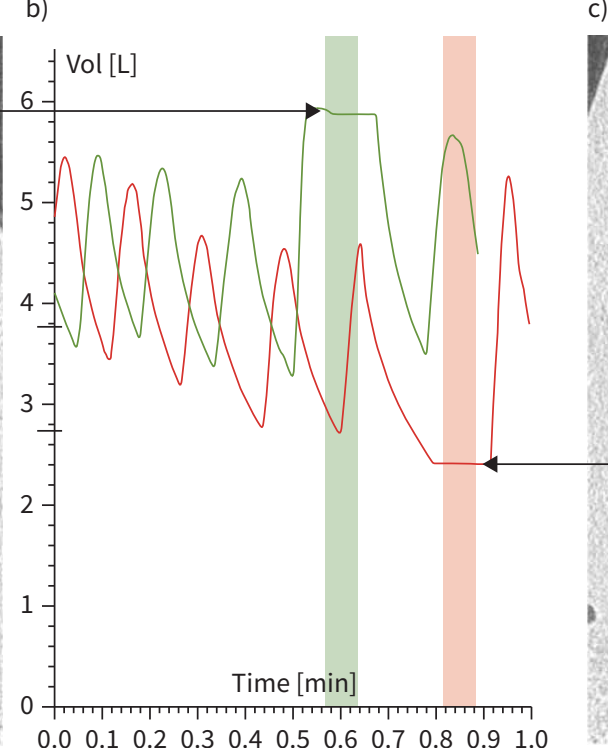

c)

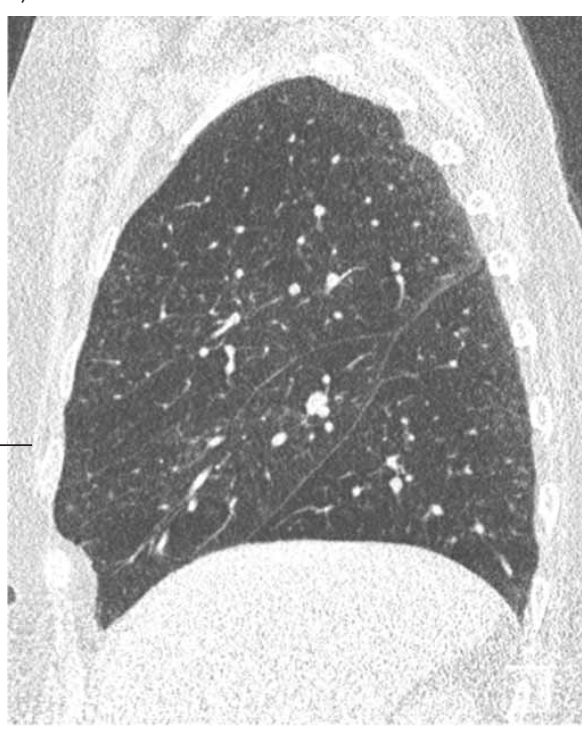

FIGURE 3 Visualisation of spirometry gating. a) Sagittal computed tomography (CT) slice of the lungs recorded in inspiration. b) Graph depicting the volumes recorded by spirometry gating over time. Green lines show inspirations and orange lines show expirations. They were recorded at separate times. Arrows from the CT images show the respective recording times. c) Sagittal CT slice of the same patient as in (a) recorded in expiration.

Figure 3 demonstrates how spirometry gating aids in CT timing. Spirometry gating with respect to lung volume estimation by CT is not yet adequately studied.

Most assessments investigating the value of spirometry-gating focused on densitometry, and not on the actual measurement of lung volumes [46-49]. This makes sense, since CT attenuation is dependent on the level of inspiration [50]. Therefore, studies investigating the impact on densitometry by means of spirometry gating give an indication of the impact spirometry gating will have on lung volume quantification.

TANTUCCI et al. [10] used spirometry gating for comparison between CT-derived TLC and plethysmography. They found a correlation factor of 0.98 and a mean difference of $0.07 \mathrm{~L}$ for obstructed patients. This seems to be an improvement compared to comparisons without use of spirometry gating. However, in this study, plethysmographic measurements were recalculated for supine positioning. CHONG et al. [38] demonstrated that CT-derived TLC measurements without spirometry gating are reproducible, suggesting that the breath holding may already be achieved quite consistently in COPD patients.

However, COPD patients mainly experience difficulty in performing complete expiration. RV is, therefore, expected to benefit the most from using spirometry gating. All studies that included a comparison between CT-derived RV and plethysmography-based RV found a weaker correlation coefficient compared to the TLC correlation coefficient. Compared to plethysmography results, studies including CT without use of spirometry gating found correlation coefficients for TLC ranging from 0.81 to 0.90 , and for RV ranging from 0.66 to 0.84 [12, 15-17].

Possible benefits from measurement of lung volumes by CT

Lung volumes determined by CT provide an opportunity to improve on the lung volume assessments now provided by plethysmography. For instance, plethysmography includes air in the body not specifically situated in the lungs, while CT is able to fully isolate the actual lung volumes.

Additionally, based on our experience, the average time to perform a CT scan is approximately $10 \mathrm{~min}$ versus 30 min for plethysmography in patients with severe COPD. Moreover, CT scans are already often made in addition to plethysmography, thus replacing plethysmography measurements by CT will improve time efficiency even further. 
Plethysmographic measurements are performed at least three times in order to establish a measurement that is as accurate as possible, whereas CT is performed once in accordance with the ALARA principle concerning radiation exposure [31]. Multiple measurements to improve precision of the measurement are preferred, however, better agreement in reproducibility has been found in CT volume measurements compared to plethysmography volume measurements [15]. A measurement without repetition is preferred from the patient's perspective. Furthermore, CT is more readily available than plethysmography in many hospitals worldwide. Although, in many hospitals, spirometry gating is not yet in use. For now, spirometry gating is mainly used in a research setting and cannot be generalised for the entire COPD population. However, in our hospital (University Medical Center Groningen, Groningen, The Netherlands) it is used for lung volume reduction and lung transplantation evaluation. This indicates that is feasible for those patients who may benefit from spirometry gating.

\section{Possible challenges in measurement of lung volumes by $\mathrm{CT}$}

Some patients are ill suited for volume measurements by CT. Patients can have difficulties with the breathing manoeuvres required to acquire a CT scan at TLC and RV. Spirometric gating may help detect patients who are not able to perform the breathing manoeuvres accurately, in addition to monitoring successfully completed and sustained breathing manoeuvres.

Some patients experience difficulty with being in a supine position. A patient in the supine position during CT is also a challenge as it may influence the lung volumes compared to the seated positioning in a non-uniform way. This means that the difference in volume acquired may be dependent on height, body mass index or other factors used to estimate a normal volume value in a complex manner. It may, therefore, not be possible to convert reliably from existing references values, which would render current standard values used in plethysmography derived volumes obsolete. Generating new reference values would require a large amount of research and seems necessary when CT-derived PFT measurements will be used on a larger scale.

Dynamic parameters: $\mathrm{FEV}_{1}$ and $D_{\mathrm{LCO}}$

The relevancy of parameters in any disease is determined by reflectiveness of the patient health status and outcome. $\mathrm{FEV}_{1}$ and $D_{\mathrm{LCO}}$ are parameters that are associated with survival [51] and, therefore, outcome surrogates used in diagnosing COPD in general, as well as in preselecting patients for LVR. FEV provides a value that is reflective of elastic recoil of the lungs and resistance of the airways, since the first second of forced exhalation is independent of the force exerted by the respiratory muscles. $D_{\text {LCO }}$ is a value reflective of the ability of the lungs to transfer oxygen in the capillary blood. Both these measurements are dynamic and therefore not obviously assessable in static CT images. However, some dynamic information may be derived from the comparison between the inspiratory and expiratory scans. Many values that can be extracted from CT images are indicative of both $\mathrm{FEV}_{1}$ and $D_{\mathrm{LCO}}$ decline, as we will discuss below.

The studies discussed below [52-63] assume, by their associations, that $\mathrm{FEV}_{1}$ and $D_{\mathrm{LCO}}$ are reproducible and representative of the severity of the disease. However, these parameters are still associated with some problems. First of all, $\mathrm{FEV}_{1}$ and $D_{\mathrm{LCO}}$ compound all lung pathology information in a single number. They can, therefore, not differentiate between types of lung pathology that may lead to differing outcomes. Secondly, both $\mathrm{FEV}_{1}$ and $D_{\mathrm{LCO}}$ are dependent on percentage predicted values to infer information on the health status of a patient.

However, the fit to a reference model of the average patient does not necessarily reflect the health status of any individual patient, since the value considered to be abnormal for the general population is not necessarily abnormal for the individual. This can be interpreted as an additional layer of noise added to the parameters of interest, namely health status and outcome. Furthermore, $\mathrm{FEV}_{1}$ is not particularly strongly related to health status [61] and is relatively insensitive to the early stages of COPD, including small airways disease [62].

\section{Densitometry-based parameters for $F E V_{1}$ and $\mathrm{D}_{L C O}$}

The first CT parameters related to COPD were density based. Emphysema noticeably leads to less dense tissue in CT, therefore being an obvious target for quantification. Emphysema is usually quantified by segmenting the lungs and calculating the percentage of voxels below a certain threshold, mean lung density or a histogram-based percentile value. Several studies have shown relationships between densitometry-based parameters and other, "more dynamic" PFT measurements, among which are FEV 1 and $D_{\text {LCO }}$ [52-55]. Densitometry emphysema scores are logically associated with $D_{\text {LCo, }}$ since destruction of lung tissue leads to a decline in gas exchange. 
Parametric response mapping for FEV ${ }_{1}$ and $\mathrm{D}_{L C O}$

There are two main pathophysiological components that contribute to COPD severity: emphysema and functional small airways disease. In an effort to separate these two components within CT, parametric response mapping (PRM) was developed in the following way. The expiration scan is mapped to the inspiration scan by use of registration. Regions $<-950 \mathrm{HU}$ in the inspiration scan and $<-856 \mathrm{HU}$ in the expiration scan are considered as emphysema $\left(\mathrm{PRM}_{\mathrm{emph}}\right)$. Regions that are $<-856 \mathrm{HU}$ in the expiration scan, but $>-950 \mathrm{HU}$ in the inspiration scan are considered functional air trapping areas $\left(\mathrm{PRM}_{\mathrm{fSAD}}\right)$ [63]. Both $\mathrm{PRM}_{\mathrm{emph}}$ and $\mathrm{PRM}_{\mathrm{fSAD}}$ are correlated with $\mathrm{FEV}_{1}$ predicted and $\mathrm{FEV}_{1}$ /forced vital capacity, as well as the transfer coefficient of the lung for carbon monoxide [64].

Airway wall thickness-based parameters for FEV ${ }_{1}$ and $\mathrm{D}_{L C O}$

In an effort to represent functional small airways disease, a variety of parameters related to airway wall dimensions, such as airway wall thickness, length and diameter were introduced. Airway wall thickness is thought to represent the inflammation present in the smaller airways. One of the parameters most commonly used is $\mathrm{Pi}_{10}$, which is the square root of the wall area of a hypothetical airway with an internal perimeter of $10 \mathrm{~mm}$ [65]. Several studies successfully related airway wall thickness-based parameters with a decline in $\mathrm{FEV}_{1}[52,56,57,66]$. A downside of using these parameters is that they are difficult to extract, since this process is not yet fully automated.

\section{Tracheal collapsibility for FEV}

Based on the reduced compliance of the airways in patients with COPD, tracheal collapsibility is frequently associated with COPD [67]. In a study by YAMASHIRO et al. [58] tracheal collapsibility was negatively correlated with $\mathrm{FEV}_{1} \%$ predicted, and positively correlated with in-/expiration lung volume ratio. In contrast, САмісіоттоц et al. [59] found a positive correlation between tracheal collapsibility and $\mathrm{FEV}_{1}$, with an additional finding that tracheal collapsibility was positively correlated with in-/expiration lung volume ratio. Sverzellati et al. [68] and Boiselle et al. [60] did not find any significant relationship between tracheal collapsibility and $\mathrm{FEV}_{1}$. Another study from Boiselle et al. [69] found a wide range of tracheal collapsibility within healthy volunteers.

All these findings seem to suggest that tracheal collapsibility might not be clinically relevant. Other airway deformity measures, such as tracheobronchial angles, however, are different in COPD patients compared to healthy individuals [70]. This is, however, more likely related to hyperinflation than to increased resistance of the airways.

\section{Discussion}

Problems with adoption of quantitative CT parameters

For a long time, different quantitative CT parameters for COPD diagnosis and evaluation have been developed and studied. However, only a few have been introduced into clinical practice. These are the parameters that provide additional information to the commonly used PFT parameters, such as densitometry parameters to signify the amount of emphysema or fissure integrity to signify the potential for collateral ventilation in LVR treatment.

Parameters that lean towards replacement of the traditional PFT parameters have generally not (yet) been introduced into clinical practice. Multiple factors are responsible for this. First, traditional PFT parameters are firmly established as the main parameters for COPD diagnosis and evaluation. This is reflected in the way quantitative CT parameters attempt to prove their worth: by association to the established PFT parameters. Even when the association is very strong, for example in the association of CT-derived TLC to PFT-derived TLC, this has not resulted in adoption into clinical practice. The traditional PFT is seen as the gold standard and it remains unclear what the impact of a switch to a CT parameter would be on the healthcare provided.

Secondly, CT is generally used in the same descriptive role it was originally intended for and therefore does not adhere to similar standardised protocols as those used in traditional PFT. Several studies found that densitometry parameters, currently in use, suffer from unstandardised CT protocols [32, 71]. Hounsfield unit thresholds are used to establish whether a voxel contains emphysema or not. The thresholds are based on comparisons with pathological assessments [72, 73], without taking into account scanning parameters that may influence the resulting outcome. For instance, it is known that the $\mathrm{kVp}$ used and the CT manufacturer has an influence on Hounsfield unit values in CT [74]. 
Thirdly, distilling meaningful quantifications from a CT dataset is difficult, since CT provides a substantial number of voxels to represent the tissue densities and precise locations of anatomical structures vary over the population. Even the most straightforward quantifications, such as lung volume estimations, require relatively complex algorithms to segment the lungs.

Additionally, while the possibility exists to derive certain parameters (semi-) automatically, such as lung volumes or densitometry measures, other parameters, such as airway wall thickness parameters and fissure integrity scores, still require more manual labour.

These factors complicate the introduction of new parameters, such as $\mathrm{PRM}, \mathrm{Pi}_{10}$ or machine learning applications, to clinical practice.

Future directives for replacement of traditional PFT by quantitative CT

There is known added value in the current descriptive role of CT use in COPD. The morphological information present on the CT is already being used as an essential part of clinical work-up for LVR. CT allows differentiating types of emphysema (e.g. centrilobular, paraseptal, panlobular) and assessing its distribution (e.g. lower lobe predominance, homogeneous). In contrast, the PFT results compound all the morphological information into one, being insensitive to the underlying morphological information.

In order to drive a movement towards more quantitative use of CT in COPD, standardisation of CT protocols is necessary. This will facilitate improved inter-observer variability and, thus, will allow sufficient comparison between facilities and help to determine stable reference values for COPD cohorts and non-COPD patients. Standardisation is, however, difficult to achieve due to a multitude of CT manufacturers, techniques, reconstruction kernels and lung segmentation algorithms. The techniques, kernels and lung segmentation algorithms are under constant development, which further complicates the standardisation. However, for any quantitative modality, differences between individual machines exist. Standardisation for CT would mean aligning settings between different machines to a maximum extent. Settings should also be maintained between different observation times.

Another method for achieving low inter-observer variability may be by use of conversion factors to a reference method. However, it is unknown whether this is feasible and this is probably less effective than standardisation of the protocols.

The replacement of traditional PFT by CT may be approached in two ways: new parameters replace the traditionally used PFT parameters or the traditional PFT parameters are mirrored by an analogous measurement on CT. In the case of RV and TLC, where there is an analogous measurement to be made on the CT, mirroring is a viable option. Since the measurement is not completely the same when done on CT, it is clear that for adoption in clinical practice future studies should focus on the impact of healthcare the provided to the patients and their outcome.

Other functional parameters without a clear analogue on CT images, such as $\mathrm{FEV}_{1}$ and $D_{\mathrm{LCO}}$, are more difficult to match. Correlation coefficients that have been found are not similar to the correlation coefficients found for lung volume estimations; it is possible that a complex combination of several parameters may be sufficient for estimating these functional parameters. For example, a partial least squares model has already been used in this way [75]. Machine learning methods, which are increasingly popular within the medical community and have already been used in the classification of COPD [76, 77], may improve upon prediction of the functional parameters through CT images [78].

When functional parameters in the future can be sufficiently predicted using machine learning models, PFT may be replaced in COPD diagnosis and evaluation for patients that already undergo a CT scan. In particular, CT appears to be inherently more sensitive than PFT parameters to early stages of COPD [62], suggesting that CT can provide more information on the status of a patient compared to PFT parameters.

For example, therefore, it is worth trying to associate CT directly to outcome with machine learning methods in an effort to establish improved diagnosis criteria based on CT.

\section{Future directives of investigation providing additional information}

CT scans performed in COPD patients contain valuable information concerning LVR preselection. LVR can be accurately planned and post-operative outcome may be predicted. For instance, it has been established that the completeness of fissures between lung lobes is an important predictor for reaching 
induced atelectasis $[79,80]$. Complete fissures do not allow for collateral ventilation between lobes. The completeness of the fissures can already be determined from CT scans by visual evaluation or quantitative algorithms [81]. Furthermore, predictions of surrogate outcome parameters, such as $\mathrm{FEV}_{1}$, based on the amount of lung volume lost by resection or atelectasis, may be made in a similar fashion as in lung cancer treatment [82-84].

Comorbidities, affecting the health status of the patient and thereby possibly influencing the LVR selection, may also be detectable. For instance, due to the relatively high contrast between pulmonary parenchyma and pulmonary vessels, the vessels can be segmented out of the lung [85]. The segmented vessels could provide useful information on pulmonary hypertension [86, 87], as pulmonary hypertension involves vascular remodelling $[88,89]$. The segmented vessels might additionally provide some indication on perfusion [90].

Quantitative parameters concerning cardiac pathologies, such as the Coronary Artery Calcium score, may also be derived. The Coronary Artery Calcium score is a predictor of cardiovascular event risk due to severe atherosclerosis [91]. Additionally, bone density assessing osteoporosis can be measured [92], providing a better indication of body fat and muscle distribution [93]. Furthermore, it has been acknowledged that body fat distribution has an influence on lung volumes [94]. Therefore another future research possibility is to investigate the influence of obesity (or body mass index) on normal lung function [95].

\section{Conclusion}

Parameters currently used in PFT are related to the pathophysiological situation of COPD, while CT is used as a descriptive modality. However, CT scans provide a detailed tissue density map of the chest allowing quantification of COPD parameters, and therefore may aid a better and easier to obtain diagnosis.

For now, quantitative CT is focused on anatomical differences in comparison to the normal physiological situation. These have been correlated to traditional PFT measurements. CT parameters that seek to replace the traditional PFT measurements have not yet been adopted in clinical practice, even though correlations of CT-derived inspiration and expiration lung volume segmentations of TLC and RV with PFT results are strong. These measurements can be improved by spirometry gating of the CT acquisitions. We expect that CT-derived analogous measurements, in particular TLC and RV, can be used in a similar fashion to traditional PFT measurements in the near future.

Dynamic measurements, such as $\mathrm{FEV}_{1}$ and $D_{\mathrm{LCO}}$, lack a clear analogous measurement in CT, and therefore mirroring is harder to achieve. Adoption of new parameters requires studies that focus on the impact of the healthcare provided. Additionally, standardisation of CT protocols should be implemented to achieve minimal inter-observer variability. This can be further exploited by machine learning methods, as these provide an opportunity to remove inherent subjectivity of multiple observers.

Provenance: Submitted article, peer reviewed.

Conflict of interest: J.T. Bakker has nothing to disclose. K. Klooster has nothing to disclose. R. Vliegenthart reports grants from Siemens Healthineers, outside the submitted work. D-J. Slebos reports grants, personal fees, non-financial support and other from PneumRX/BTG, USA/UK and PulmonX, USA, and grants, personal fees and non-financial support from CSA Medical, USA, and Nuvaira, USA, outside the submitted work.

\section{References}

1 World Health Organization. The top 10 causes of death. www.who.int/news-room/fact-sheets/detail/ the-top-10-causes-of-death Date last updated: 9 December 2020.

2 Teschler $\mathrm{H}$, Stamatis G, El-Raouf Farhat AA, et al. Effect of surgical lung volume reduction on respiratory muscle function in pulmonary emphysema. Eur Respir J 1996; 9: 1779-1784.

3 Global Initiative for Chronic Obstructive Lung Disease. Global strategy for the diagnosis, management, and prevention of chronic obstructive pulmonary disease. 2020 report. https://goldcopd.org/gold-reports/

4 Huang WM, Feng JY, Cheng HM, et al. The role of pulmonary function in patients with heart failure and preserved ejection fraction: looking beyond chronic obstructive pulmonary disease. PLoS One 2020; 15: e0235152.

5 van Dijk M, Klooster K, Ten Hacken NHT, et al. The effects of lung volume reduction treatment on diffusing capacity and gas exchange. Eur Respir Rev 2020; 29: 190171.

6 van Geffen WH, Slebos DJ, Herth FJ, et al. Surgical and endoscopic interventions that reduce lung volume for emphysema: a systemic review and meta-analysis. Lancet Respir Med 2019; 7: 313-324. 
7 Shah PL, Herth FJ, van Geffen WH, et al. Lung volume reduction for emphysema. Lancet Respir Med 2017; 5: $147-156$.

8 Hartman JE, Vanfleteren LEGW, van Rikxoort EM, et al. Endobronchial valves for severe emphysema. Eur Respir Rev 2019; 28: 180121.

9 Slebos DJ, Shah PL, Herth FJF, et al. Endobronchial valves for endoscopic lung volume reduction: best practice recommendations from expert panel on endoscopic lung volume reduction. Respiration 2017; 93: 138-150.

10 Tantucci C, Bottone D, Borghesi A, et al. Methods for measuring lung volumes: is there a better one? Respiration 2016; 91: 273-280.

11 Garfield JL, Marchetti N, Gaughan JP, et al. Total lung capacity by plethysmography and high-resolution computed tomography in COPD. Int J COPD 2012; 7: 119-126.

12 Becker MD, Berkmen YM, Austin JHM, et al. Lung volumes before and after lung volume reduction surgery: quantitative CT analysis. Am J Respir Crit Care Med 1998; 157: 1593-1599.

13 Coxson HO, Nasute Fauerbach PV, Storness-Bliss C, et al. Computed tomography assessment of lung volume changes after bronchial valve treatment. Eur Respir J 2008; 32: 1443-1450.

14 Matsumoto AJ, Bartholmai BJ, Wylam ME. Comparison of total lung capacity determined by plethysmography with computed tomographic segmentation using CALIPER. J Thorac Imaging 2017; 32 101-106.

15 Brown MS, Kim HJ, Abtin F, et al. Reproducibility of lung and lobar volume measurements using computed tomography. Acad Radiol 2010; 17: 316-322.

16 Kauczor HU, Heussel CP, Fischer B, et al. Assessment of lung volumes using helical CT at inspiration and expiration: comparison with pulmonary function tests. Am J Roentgenol 1998; 171: 1091-1095.

17 Zaporozhan J, Ley S, Eberhardt R, et al. Paired inspiratory/expiratory volumetric thin-slice CT scan for emphysema analysis: comparison of different quantitative evaluations and pulmonary function test. Chest 2005; 128: 3212-3220.

18 Brown MS, McNitt-Gray MF, Goldin JG, et al. Automated measurement of single and total lung volume from CT. J Comput Assist Tomogr 1999; 23: 632-640.

19 Song L, Leppig JA, Hubner RH, et al. Quantitative CT analysis in patients with pulmonary emphysema: do calculated differences between full inspiration and expiration correlate with lung function? Int J COPD 2020 15: $1877-1886$.

20 Miller MR, Crapo R, Hankinson J, et al. General considerations for lung function testing. Eur Respir J 2005; 26: 153-161.

21 Coates AL, Peslin R, Rodenstein D, et al. Measurement of lung volumes by plethysmography. Eur Respir J 1997; 10: 1415-1427.

22 Wanger J, Clausen JL, Coates A, et al. Standardisation of the measurement of lung volumes. Eur Respir J 2005; 26: 511-522.

23 Criée CP, Sorichter S, Smith HJ, et al. Body plethysmography - Its principles and clinical use. Respir Med 2011; 105: 959-971.

24 Reinert M, Trendelenburg F. Advantage of simultaneous bodyplethysmographic and spirographic recording. Pneumonologie 1971; 146: 79-82.

25 Welling JBA, Hartman JE, Ten Hacken NHT, et al. Significant differences in body plethysmography measurements between hospitals in patients referred for bronchoscopic lung volume reduction. Lung 2019; 197: 573-576.

26 Williams JH, Bencowitz HZ. Differences in plethysmographic lung volumes. Effects of linked vs unlinked spirometry. Chest 1989; 95: 117-123.

27 Shore SA, Huk O, Mannix S, et al. Effect of panting frequency on the plethysmographic determination of thoracic gas volume in chronic obstructive pulmonary disease. Am Rev Respir Dis 1983; 128: 54-59.

28 Newth CJL, Enright P, Johnson RL. Multiple-breath nitrogen washout techniques: Including measurements with patients on ventilators. Eur Respir J 1997; 10: 2174-2185.

29 Publications Office of the European Commission. European guidelines on quality criteria for computed tomography. EUR 16262. https://op.europa.eu/en/publication-detail/-/publication/d229c9e1-a967-49de-b16959ee68605f1a Date last updated: 18 February 2020.

30 Mayo JR. CT evaluation of diffuse infiltrative lung disease: dose considerations and optimal technique. $J$ Thorac Imaging 2009; 24: 252-259.

31 The 2007 Recommendations of the International Commission on Radiological Protection. ICRP publication 103. Ann ICRP 2007; 37: 1-332.

32 Gierada DS, Bierhals AJ, Choong CK, et al. Effects of CT section thickness and reconstruction kernel on emphysema quantification. Relationship to the Magnitude of the CT Emphysema Index. Acad Radiol 2010; 17: 146-156.

33 Xu M, Qi S, Yue Y, et al. Segmentation of lung parenchyma in CT images using CNN trained with the clustering algorithm generated dataset. Biomed Eng Online 2019; 18: 2. 
34 Mansoor A, Bagci U, Foster B, et al. Segmentation and image analysis of abnormal lungs at CT: current approaches, challenges, and future trends. Radiographics 2015; 35: 1056-1076.

35 Mansoor A, Bagci U, Xu Z, et al. A Generic approach to pathological lung segmentation. IEEE Trans Med Imaging 2014; 33: 2293-2310.

36 Van Rikxoort EM, De Hoop B, Viergever MA, et al. Automatic lung segmentation from thoracic computed tomography scans using a hybrid approach with error detection. Med Phys 2009; 36: 2934-2947.

37 Shen M, Tenda ED, McNulty W, et al. Quantitative evaluation of lobar pulmonary function of emphysema patients with endobronchial coils. Respiration 2019; 98: 70-81.

38 Chong D, Brown MS, Kim HJ, et al. Reproducibility of volume and densitometric measures of emphysema on repeat computed tomography with an interval of 1 week. Eur Radiol 2012; 22: 287-294.

39 Chen F, Kubo T, Shoji T, et al. Comparison of pulmonary function test and computed tomography volumetry in living lung donors. J Heart Lung Transplant 2011; 30: 572-575.

40 Iwano S, Okada T, Satake H, et al. 3D-CT volumetry of the lung using multidetector row $\mathrm{CT}$. Comparison with pulmonary function tests. Acad Radiol 2009; 16: 250-256.

41 Yamada Y, Yamada M, Chubachi S, et al. Comparison of inspiratory and expiratory lung and lobe volumes among supine, standing, and sitting positions using conventional and upright CT. Sci Rep 2020; 10: 16203 .

42 Jung WS, Haam S, Shin JM, et al. The feasibility of CT lung volume as a surrogate marker of donor-recipient size matching in lung transplantation. Medicine 2016; 95: e3957.

43 Blair E, Hickam JB. The effect of change in body position on lung volume and intrapulmonary gas mixing in normal subjects. J Clin Invest 1955; 34: 383-389.

44 Allen SM, Hunt B, Green M. Fall in vital capacity with posture. Br J Dis Chest 1985; 79: 267-271.

45 Fromageot $\mathrm{C}$, Lofaso $\mathrm{F}$, Annane $\mathrm{D}$, et al. Supine fall in lung volumes in the assessment of diaphragmatic weakness in neuromuscular disorders. Arch Phys Med Rehabil 2001; 82: 123-128.

46 Kalendar WA, Rienmuller R, Seissler W, et al. Measurement of pulmonary parenchymal attenuation: use of spirometric gating with quantitative CT. Radiology 1990; 175: 265-268.

47 Gierada DS, Yusen RD, Pilgram TK, et al. Repeatability of quantitative CT indexes of emphysema in patients evaluated for lung volume reduction surgery. Radiology 2001; 220: 448-454.

48 Moroni C, Mascalchi M, Camiciottoli G, et al. Comparison of spirometric-gated and -ungated HRCT in COPD. J Comput Assist Tomogr 2003; 27: 375-379.

49 Otjen JP, Swanson JO, Oron A, et al. Spirometry-assisted high resolution chest computed tomography in children: is it worth the effort? Curr Probl Diagn Radiol 2018; 47: 14-18.

50 Robinson PJ, Kreel L. Pulmonary tissue attenuation with computed tomography: comparison of inspiration and expiration scans. J Comput Assist Tomogr 1979; 3: 740-748

51 Neas LM, Schwartz J. Pulmonary function levels as predictors of mortality in a national sample of US adults. Am J Epidemiol 1998; 147: 1011-1018.

52 Mohamed Hoesein FAA, de Jong PA, Lammers JWJ, et al. Contribution of CT quantified emphysema, air trapping and airway wall thickness on pulmonary function in male smokers with and without COPD. COPD 2014; 11: 503-509.

53 Schroeder JD, McKenzie AS, Zach JA, et al. Relationships between airflow obstruction and quantitative CT measurements of emphysema, air trapping, and airways in subjects with and without chronic obstructive pulmonary disease. Am J Roentgenol 2013; 201: 460-470.

54 Heremans A, Verschakelen JA, Van Fraeyenhoven L, et al. Measurement of lung density by means of quantitative CT scanning; a study of correlations with pulmonary function tests. Chest 1992; 102: 805-811.

55 Klein JS, Gamsu G, Webb WR, et al. High-resolution CT diagnosis of emphysema in symptomatic patients with normal chest radiographs and isolated low diffusing capacity. Radiology 1992; 182: 817-821.

56 Dijkstra AE, Postma DS, ten Hacken N, et al. Low-dose CT measurements of airway dimensions and emphysema associated with airflow limitation in heavy smokers: a cross sectional study. Respir Res 2013; 14: 11.

57 Mohamed Hoesein FAA, De Jong PA, Lammers JWJ, et al. Airway wall thickness associated with forced expiratory volume in $1 \mathrm{~s}$ decline and development of airflow limitation. Eur Respir J 2015; 45: 644-651.

58 Yamashiro T, San José Estépar R, Matsuoka S, et al. Intrathoracic tracheal volume and collapsibility on inspiratory and end-expiratory CT Scans. Correlations with lung volume and pulmonary function in 85 smokers. Acad Radiol 2011; 18: 299-305.

59 Camiciottoli G, Diciotti S, Bigazzi F, et al. Is intrathoracic tracheal collapsibility correlated to clinical phenotypes and sex in patients with COPD? Int J COPD 2015; 10: 843-852.

60 Boiselle PM, Michaud G, Roberts DH, et al. Dynamic expiratory tracheal collapse in COPD: correlation with clinical and physiologic parameters. Chest 2012; 142: 1539-1544.

61 Jones PW. Health status and the spiral of decline. COPD 2009; 6: 59-63.

62 Koo HK, Vasilescu DM, Booth S, et al. Small airways disease in mild and moderate chronic obstructive pulmonary disease: a cross-sectional study. Lancet Respir Med 2018; 6: 591-602.

63 Galbán CJ, Han MK, Boes JL, et al. Computed tomography-based biomarker provides unique signature for diagnosis of COPD phenotypes and disease progression. Nat Med 2012; 18: 1711-1715. 
64 Pompe E, Galbán CJ, Ross BD, et al. Parametric response mapping on chest computed tomography associates with clinical and functional parameters in chronic obstructive pulmonary disease. Respir Med 2017; 123: 48-55.

65 Nakano Y, Wong JC, De Jong PA, et al. The prediction of small airway dimensions using computed tomography. Am J Respir Crit Care Med 2005; 171: 142-146.

66 Ohara T, Hirai T, Sato S, et al. Longitudinal study of airway dimensions in chronic obstructive pulmonary disease using computed tomography. Respirology 2008; 13: 372-378.

67 Murgu SD, Colt HG. Tracheobronchomalacia and excessive dynamic airway collapse. Respirology 2006; 11: 388-406.

68 Sverzellati N, Rastelli A, Chetta A, et al. Airway malacia in chronic obstructive pulmonary disease: prevalence, morphology and relationship with emphysema, bronchiectasis and bronchial wall thickening. Eur Radiol 2009; 19: 1669-1678.

69 Boiselle PM, Donnell CRO, Bankier AA, et al. Tracheal collapsibility in healthy volunteers during forced expiration: assessment with multidetector CT. Radiology 2009; 252: 255-262.

70 Onoe R, Yamashiro T, Handa H, et al. 3D-measurement of tracheobronchial angles on inspiratory and expiratory chest CT in COPD: respiratory changes and correlation with airflow limitation. Int J COPD 2018; 13 : 2399-2407.

71 Kemerink GJ, Kruize HH, Lamers RJS, et al. CT lung densitometry: dependence of CT number histograms on sample volume and consequences for scan protocol comparability. J Comput Assist Tomogr 1997; 21: 948-954.

72 Muller NL, Staples CA, Miller RR, et al. "Density mask". An objective method to quantitate emphysema using computed tomography. Chest 1988; 94: 782-787.

73 Gould GA, MacNee W, McLean A, et al. CT measurements of lung density in life can quantitate distal airspace enlargement: an essential defining feature of human emphysema. Am Rev Respir Dis 1988; 137: 380-392.

74 Cropp RJ, Seslija P, Tso D, et al. Scanner and kVp dependence of measured CT numbers in the ACR CT phantom. J Appl Clin Med Phys 2013; 14: 338-349.

75 Gu S, Leader J, Zheng B, et al. Direct assessment of lung function in COPD using CT densitometric measures. Physiol Meas 2014; 35: 833-845.

76 Bermejo-Pelaez D, Estépar RSJ, Ledesma-Carbayo MJ. Emphysema classification using a multi-view convolutional network. Proc IEEE Int Symp Biomed Imaging 2018; 2018: 519-522.

77 Humphries SM, Notary AM, Centeno JP, et al. Deep learning enables automatic classification of emphysema pattern at CT. Radiology 2020; 294: 434-444.

78 Gawlitza J, Sturm T, Spohrer K, et al. Predicting pulmonary function testing from quantified computed tomography using machine learning algorithms in patients with COPD. Diagnostics 2019; 9: 33.

79 Herth FJF, Noppen M, Valipour A, et al. Efficacy predictors of lung volume reduction with Zephyr valves in a European cohort. Eur Respir J 2012; 39: 1334-1342.

80 Valipour A, Herth FJF, Burghuber OC, et al. Target lobe volume reduction and COPD outcome measures after endobronchial valve therapy. Eur Respir J 2014; 43: 387-396.

81 Klooster K, David Koster T, Ruwwe-Glösenkamp C, et al. An integrative approach of the fissure completeness score and chartis assessment in endobronchial valve treatment for emphysema. Int J COPD 2020; 15: 1325-1334.

82 Fourdrain A, De Dominicis F, Lafitte S, et al. Quantitative computed tomography to predict postoperative FEV1 after lung cancer surgery. J Thorac Dis 2017; 9: 2413-2418.

83 Wu M-T, Pan H-B, Chiang AA, et al. Prediction of postoperative lung function in patients with lung cancer. Am J Roentgenol 2002; 178: 667-672.

84 Ohno $\mathrm{Y}$, Koyama $\mathrm{H}$, Nogami $\mathrm{M}$, et al. Postoperative lung function in lung cancer patients: comparative analysis of predictive capability of MRI, CT, and SPECT. Am J Roentgenol 2007; 189: 400-408.

85 Zhai Z, Staring M, Hernández Girón I, et al. Automatic quantitative analysis of pulmonary vascular morphology in CT images. Med Phys 2019; 46: 3985-3997.

86 Aluja Jaramillo F, Gutierrez FR, Díaz Telli FG, et al. Approach to pulmonary hypertension: from CT to clinical diagnosis. Radiographics 2018; 38: 357-373.

87 Coste F, Benlala I, Dournes G, et al. Assessing pulmonary hypertension in COPD. Is there a role for computed tomography? Int J COPD 2019; 14: 2065-2079.

88 Magee F, Wright JL, Wiggs BR, et al. Pulmonary vascular structure and function in chronic obstructive pulmonary disease. Thorax 1988; 43: 183-189.

89 Tuder RM. Pulmonary vascular remodeling in pulmonary hypertension. Cell Tissue Res 2017; 367: 643-649.

90 Koster TD, Hazrati E, Van Rikxoort EM, et al. CT-approximated perfusion is comparable to nuclear perfusion imaging. Eur Respir J 2020; 56: Suppl. 64, 1575.

91 Greenland P, Blaha MJ, Budoff MJ, et al. Coronary calcium score and cardiovascular risk. J Am Coll Cardiol 2018; 72: 434-447. 
92 Gausden EB, Nwachukwu BU, Schreiber JJ, et al. Opportunistic use of CT imaging for osteoporosis screening and bone density assessment. J Bone Joint Surg Am 2017; 99A: 1580-1590.

93 Sanders KJC, Klooster K, Vanfleteren LEGW, et al. CT-derived muscle remodelling after bronchoscopic lung volume reduction in advanced emphysema. Thorax 2019; 74: 206-207.

94 Collins LC, Hoberty PD, Walker JF, et al. The effect of body fat distribution on pulmonary function tests. Chest 1995; 107: 1298-1302.

95 Littleton SW. Impact of obesity on respiratory function. Respirology 2012; 17: 43-49. 